\title{
A rare case of oncocytic Schneiderian Papilloma with intradural and intraorbital extension with notes of operative techniques*
}

\author{
Maurizio Bignami ${ }^{1}$, Andrea Pistochini ${ }^{1}$, Francesco Meloni ${ }^{2}$, Emilio Delehaye $^{2}$, \\ Paolo Castelnuovo ${ }^{1}$ \\ Department of Otorhinolaryngology, University of Insubria, Azienda Ospedaliera-Universitaria, Ospedale di \\ Circolo e Fondazione Macchi, Varese, Italy \\ 2 Department of Otorhinolaryngology, University of Sassari, Azienda Ospedaliera-Universitaria, Sassari, Italy
}

\begin{abstract}
SUMMARY
Epithelial cells of cylindrical cell papilloma are oncocytes, which arise from the sinonasal respiratory epithelium, hence the term Oncocytic Schneiderian papilloma.This is a rare and benign neoplasm of the nose and paranasal sinuses and it should be considered in the work-up of all unilateral nasal polypoid lesions. Clinically behaviour is comparable to inverted papillomas for local recurrence and malignancy coexistence.

We report a case arisen from the nasoethmoidal space that extended to the anterior skull base through a bone dehiscence with intradural invasion and orbital space involvement. Surgical therapy is the treatment of choice, the endonasal endoscopic approach can be used in most of the cases and this surgical technique is safe and suitable also in presence of an extra nasal extension. We describe our experience for management of this kind of lesions and some notes on our operative technique.
\end{abstract}

Key words: Schneiderian, papilloma, intradural, intraorbital, technique

\section{INTRODUCTION}

Barnes and Bedetti ${ }^{(1)}$ demonstrated that the epithelial cells of the cylindrical cell papilloma are the oncocytes which arise from the sinonasal respiratory epithelium, hence the term Oncocytic Schneiderian papilloma (OSP). OSP, fungiform papilloma and inverted papilloma are three morphologically different tumours arising from the Schneiderian membrane. OSP comprises approximately $3 \%$ to $5 \%$ of this entity and it is the rarest one. Old-aged group predominates and no sex predilection is noted ${ }^{(2)}$. Therefore OSP is a rare and benign neoplasm of the nose and paranasal sinuses and it should be considered in the work-up of all unilateral nasal polypoid lesions. This entity generally does not show a tendency to invade locally, but only a compressive effect, during its growth, on the adjacent structures.

Clinically behavior is comparable to inverted papillomas for local recurrence and malignancy coexistence. Malignancies arising in OSPs are an extremely rare event ${ }^{(3)}$. The recurrence rate for OSP is $33 \%{ }^{(4)}$.

We reported an OSP case arising from the nasoethmoidal space and extending to anterior skull base through a bone dehiscence with intradural invasion and orbital space involvement. To our knowledge this is the only case reported in the literature with a concomitant endocranial and orbital extension.

Surgical therapy is the treatment of choice, endonasal endoscopic approach can be used in most of the cases and this surgical technique is safe and suitable also in presence of extranasal extension.

\section{CASE REPORT}

A 71-year-old woman had a 6-month history of progressive right-sided nasal obstruction. Her awareness of the presence of a growth in her right nostril, over the previous several weeks, prompted her to seek medical evaluation.

She denied epistaxis, rhinorrhea, headaches, ophthalmic and neurological symptoms. Physical examination revealed the presence of a polypoid mass which partially filled the right nostril without extension into the nasal vestibule. The size of the mass prevented exact localization of its origin from the lateral nasal wall, in the region of middle and superior meatus. The left nasal fossa was free from disease. Septal mucosa and nasopharyngeal space were not involved. The remainder of the head and neck and general examination revealed no abnormalities. 


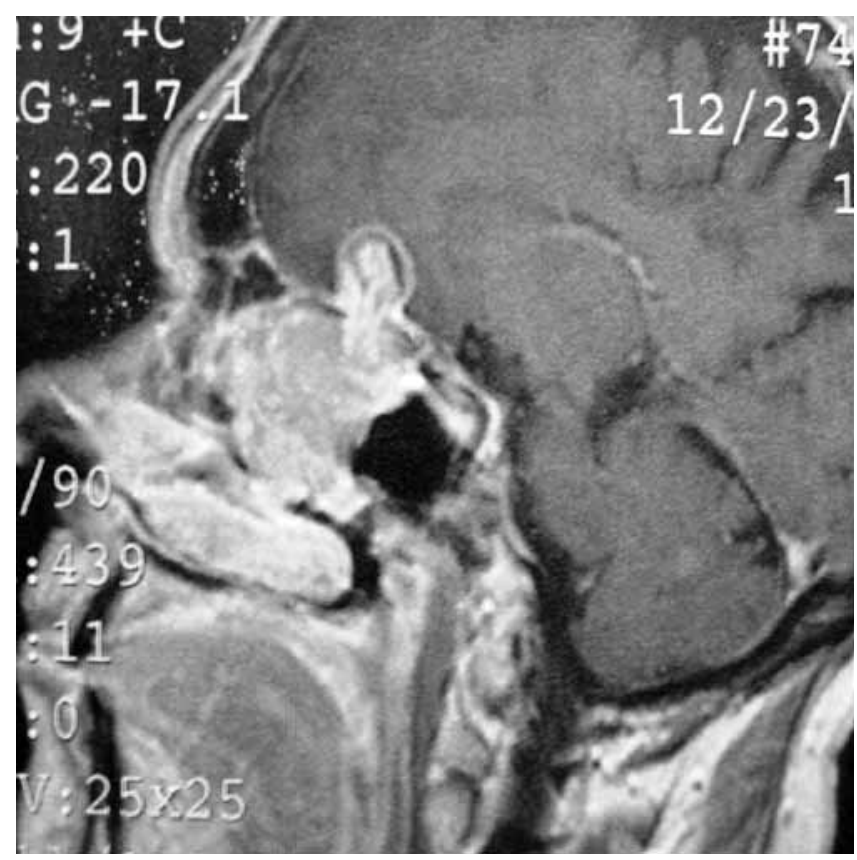

Figure 1. Preoperative sagittal contrast T1-weighted MR view in sagittal plane: hyperintense enhancing nasal and ethmoidal mass invading the anterior cranial fossa through posterior right frontoetmhmoidal bone. Peripheral well defined borders with frontal inferior gyral simple displacement.

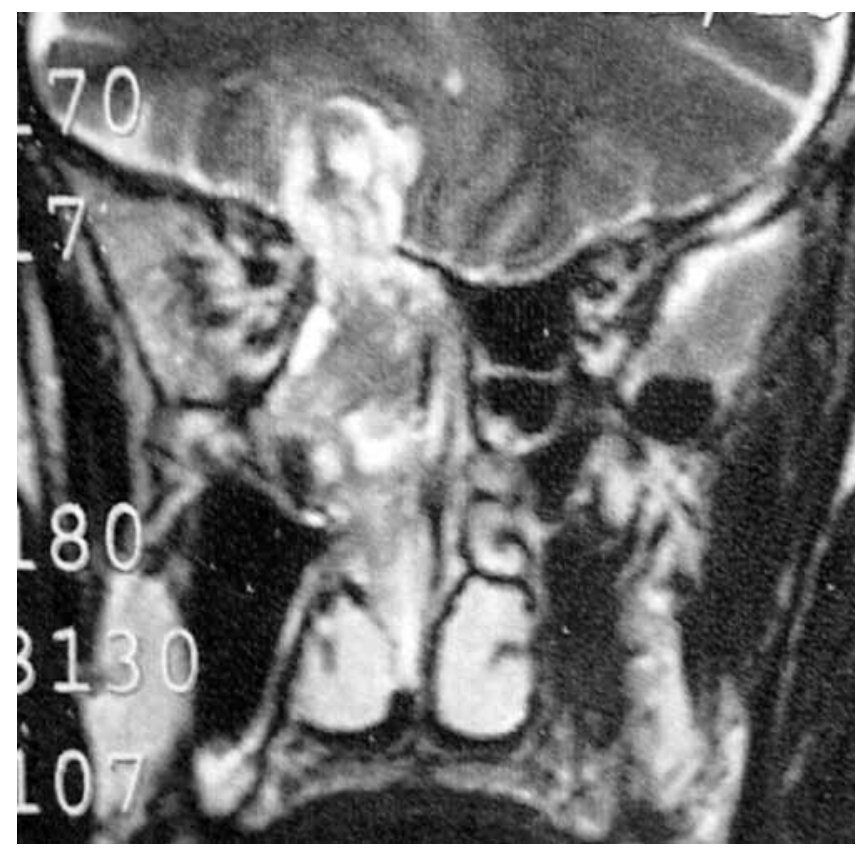

Figure 2. Preoperative MR coronal T2-weighted view of the mass extending from middle-upper right nasal fossa to the ethmoidal cavities and anterior cranial fossa. Apparent medial orbit wall erosion with no periorbital infiltration. The internal signal is etherogeneous due to stromal and epithelial intensities assuming the typical "cerebriform" pattern of inverted papilloma.
A computed tomographic scan of the neck, chest and abdomen and total body bone scintigraphy were negative for secondaries. CT evaluation of rhino-sinusal area showed a large lesion in the right nasal fossa, which involved the middle and superior turbinate but not the nasal septum. Bone erosion was also evident, particularly in the posterior ethmoidal compartment and lamina papiracea. Medial rectus muscle appeared displaced by the lesion and skull base was eroded with frontal lobe intradural invasion.

MR was used for a better definition of the extent beyond nasal cavity. T1 sequences after contrast administration, in sagittal and coronal planes, revealed an hyperintense, enhancing nasal and ethmoidal mass invading the anterior cranial fossa through posterior right fronto-etmhmoidal bone. Medial rectus muscle was confirmed to be laterally displaced but not involved by the lesion (Figures 1 and 2). Pre-operative biopsies were endoscopically performed in three different sites: posterior ethmoid, middle meatus and above the right choanal aspect. The excised tissue consisted of soft fragments and specimens were submitted for histopathologic processing.

Histopathological assessment of sampled tissue revealed the presence of an oncocytic papilloma. After diagnostic work-up the nasal papilloma was treated with endonasal endoscopic resection technique ${ }^{(5,6)}$.

Ethmoidal compartment was resected with laser assisted subperiosteal centripetal technique. This technique allowed us to remove the nasosinusal mass and its extension to the anterior skull base, keeping some essentials steps:

- central tumor debulking (piecemeal removal);

- subperiosteal dissection including all the ethmoid. First incision, horseshoe fashioned, included nasal septum, nasal cavity roof anteriorly to the first olfactory fibers, lamina papiracea and lateral nasal wall. This surgical method allowed us the centripetal, antero-posterior undermining of a single flap of periosteum, including phatological tissue;

- maxillary sinus medial wall and lamina papyracea were excised, while cribriform plate, nasal septum and unimpaired ethmoidal roof were preserved;

- the intraorbital part of papilloma was resected, while periorbital tissue was regular and then preserved after periorbit frozen samples were analyzed.

Subsequently the skull base defect in posterior ethmoids was identified and was enlarged by drilling so as to allow the resection of the endocranial part of lesion. During this step multiple frozen biopsies on the intracranial tissue were performed and they showed normal cerebral tissue.

Finally skull base duraplasty in a multilayer (three layer) fashion was performed: after having drilled the bone around the defect and at the level of the basal sheet, to obtain a smooth and regular surface, an intracranial intradural free graft of synthetic dural substitute (Neuropatch ${ }^{\circledR}$, Aesculap, Tuttlingen, 


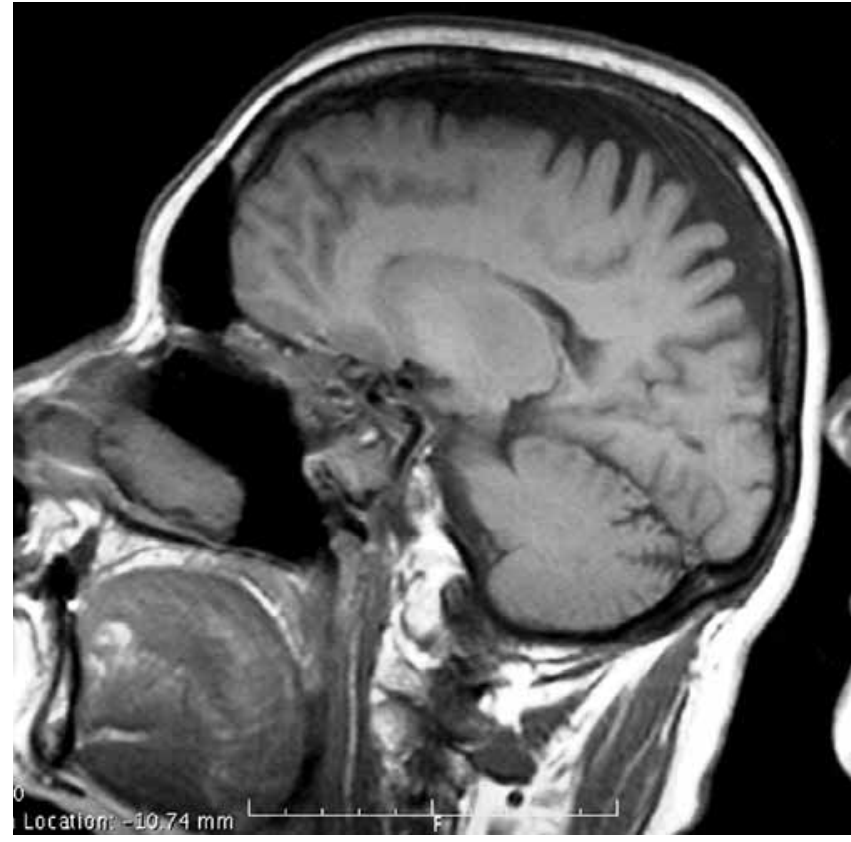

Figure 3. Postoperative unenhanced sagittal T1-w image showing sinonasal and anterior cranial fossa mass removal with wall repair.

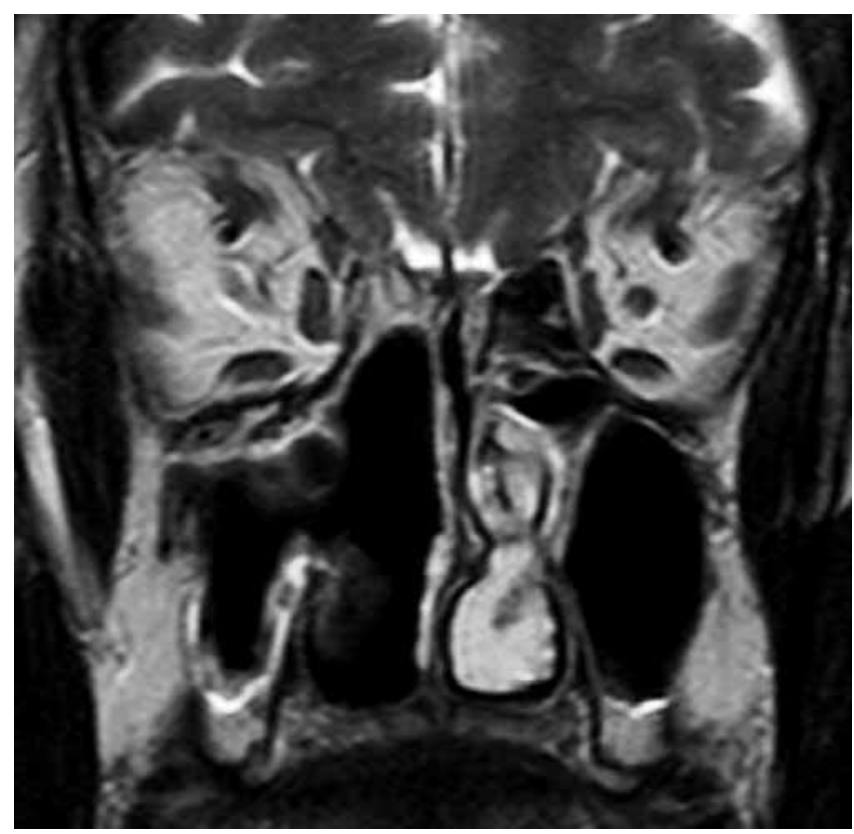

Figure 4. Postoperative T2-weighted coronal view with no residual lesion visible.

Germany) and an intracranial extradural septal cartilage free graft were positioned with underlay technique to give increased support to intracranial content. Septal mucoperiosteum graft was placed, with overlay technique, on the nasal side and then fixed with fibrin's glue and Oxicel ${ }^{\circledR}$ (Johnson \& Johnson Medical Ltd, North Yorkshire, UK) sponges.

All the other paranasal sinuses explored had normal features. During operation many frozen biopsies were performed on surrounding mucosa and they were all negative for malignant foci and papilloma.
No intraoperative and postoperative complications were registered and no evidence of malignant disease in definitive pathological examination was found. Endoscopic endonasal controls were performed at 15-30-60-90 days after surgery and then every 6 months. MR control was scheduled after 6 months and than repeated every 6 months for the first 2 years, than once per year.

Endoscopic controls showed a normal nasal mucosa healing, no orbital fat herniation and wide paranasal sinusotomies. Ethmoidal roof duraplasty showed well positioned and vital intranasal graft without evidence of a CSF leak or disease recurrence.

Postoperative MRI, after three years, showed a good result with sino-nasal and anterior cranial fossa mass removal and correct duraplasty (Figures 3 and 4). After nearly three years of follow-up the patient is completely free for disease.

\section{DISCUSSION}

Removal of this kind of lesion is now performed mainly in tertiary referal centres. An intracranial extension of such a lesion is unusual and the diagnosis and treatment modalities are not definitively clear.

Because of the high rate of recurrence or persistence after insufficient surgical excision, and considering the possibility of missing an associated carcinoma in inadequate biopsy, a medial maxillectomy through a lateral rhinotomy with en bloc resection of the lateral nasal wall in the past was established as the gold standard for management of these lesions ${ }^{(7)}$.

Nowadays many authors prefer an endonasal endoscopic approach as useful, safe and minivasive technique ${ }^{(8)}$. In the hand of experienced and skilled surgeons, complete endoscopic removal is attainable in most cases. En bloc resection is not necessary to achieve oncologic cure ${ }^{(9)}$. Anyway it is extremely difficult to compare the results obtained with external versus endoscopic or microendoscopic approaches because of the extreme variability in preoperative assessment, extent of disease, and length of follow-up in the different reported series.

Our experience ${ }^{(6)}$ concerning the treatment of inverted papilloma shows better results with an endoscopic endonasal approach allowing us a multi-angled, magnified view of the surgical field and an optimal chance to differentiate between normal and diseased mucosa. As we have demonstrated in a previous manuscript ${ }^{(6)}$, the extent of dissection can be tailored to the extent of disease to include a large spectrum of lesions, from those confined to middle meatus to those extensively involving the maxillary sinus or surrounding anatomical structures. For different extensions of the lesion we suggest 3 different types of endonasal maxillectomy of increasing surgical aggressiveness. As shown in our results ${ }^{(6)}$ en bloc resection is not a prerequisite to achieve a radical resection. Whereas in our opinion, the most important principle is the diseased mucosa dissection along the subperiosteal plane, drilling the underlying bone whenever required by radiological and intra- 
operative findings. En bloc resection is certainly feasible for lesions limited to the middle meatus and to nasal cavity but can be rarely achieved in case of neoplasms entirely filling the nasal cavity or extending toward orbit and cranial base. It is our practice to verify the radicality of the resection by frozen sections and to separately evaluate surgical specimens in patients not amenable to en bloc resection for definitive histology.

The possibility of missing an associated carcinoma is very low and acceptable ${ }^{(10)}$, as with conventional technique, if a meticulous removal of all additional mucosa in the ipsilateral paranasal sinuses is realized. Nevertheless the eventuality of an intracranial and intraorbital extension of this kind of benign neoplasm was never taken into consideration. The lesions, as in this case, which presents with an endocranial and intraorbital extension, can be also safely treated with an endonasal endoscopic resection. Since the removal of the endocranial part of the lesion could implicate a risk for endocranial bleeding, some anatomical considerations and a careful surgical planning (with the neurosurgeon) should be done before surgery to avoid and to possibly be able to manage this complication.

Patients should always be informed during preoperative counselling about the possibility of reverting the operation to an external approach. The surgical procedure must be performed under such circumstances that the surgical techniques are ready to be modified when needed and to possibly quickly manage haemorrhagic complications. In case of intracranial invasion an MR study is necsessary in order to evaluate neoplasm's features, presence of pial adherence and presence of close blood vessels. Surgeon's skill is important in gently treating the intracranial part of the lesion. Availability for frozen sections to evaluate margins and endocranial tissue is essential. Observing these guidances excision can be safe, avoiding a more invasive approach and possibly a loss of the sense of smell. Overall it seemed to guarantee a long disease-free period, until three years as in our report. Prevention remains elusive, because the origin of the OSP is unknown. Viral infection, allergy, chronic inflammation, smoking, and other environmental carcinogens have all been cited as possible contributors with no supportive data.

\section{REFERENCES}

1. Barnes L, Bedetti C. Oncocytic Schneiderian paillomas: a reappraisal of cylindrical cell papilloma of the sinonasal tract. Hum Pathol 1984; 15: 344-351.

2. Dominguez OB, Colina MV, Ramirez Ruiz RD, et al. Papiloma Schneideriano de celulas oncociticas. Anales ORL Iber-Amer. XXXII 2005; 2: 115-123.

3. Maitra A, Baskin LB, Lee EL. Malignancies Arising in Oncocytic Schneiderian Papillomas. Arch Pathol Lab Med 2001; 125: 13651367.

4. Kaufman MR, Branwein MS, Lawson W. Sinonasal Papillomas: Clinicopathological review of 40 patients with inverted and oncocytic Schneiderian papillomas. Laryngoscope 2002; 112: 1372-7.

5. Paolo Castelnuovo, Paolo Battaglia, Davide Locatelli, Giovanni Delù, Federica Sberze, Maurizio Bignami. Endonasal micro-endoscopic treatment of malignant tumors of the paranasal sinuses and anterior skull base. Operative Techn Otolaryngol 2006; 17: 152-167.

6. Tomenzoli D, Castelnuovo P, Pagella F, Berlucchi M, Pianta L, Delu G, Maroldi R, Nicolai P. Different endoscopic surgical strategies in the management of inverted papilloma of the sinonasal tract: experience with 47 patients. Laryngoscope. 2004; 114: 193200.

7. Cunningham MJ, Brantley S, Barnes L, Schramm VL Jr. Oncocityc Schneiderian papilloma in a young adult: a rare diagnosis. Otolaryngol Head Neck Surg 1987; 97: 47-51.

8. Minovi A, Kollert M, Draf W, Bockmühl U. Inverted papilloma: feasibility of endonasal surgery and long-term results of 87 cases. Rhinology. 2006; 44: 205-210.

9. Banhiran W, Casiano RR. Endoscopic sinus surgery for benign and malignant nasal and sinus neoplasm. Curr Opin Otolaryngol Head Neck Surg. 2005 Feb;13(1):50-4.

10. Udaka T, Shiomori T, Nagatani G, Hisaoka M, Kakeda S, Korogi Y, Suzuki H. Oncocytic schneiderian papilloma confined to the sphenoid sinus detected by FDG-PET. Rhinology. 2007; 45: 89-92.

\author{
Emilio Delehaye \\ Department of Otorhinolaryngology \\ University of Sassari \\ Azienda Ospedaliera-Universitaria \\ via Cavour 25 \\ 07100 Sassari \\ Italy \\ Tel: $+39-79-228509$ \\ Fax: +39-79-22 8060 \\ E-mail: entsassari@tiscali.it; delemy@tiscali.it.
}

